\title{
Role of corpectomy in cervical spondylosis
}

\author{
IAIN H. KALFAS, M.D. \\ Department of Neurosurgery, Section of Spinal Surgery, The Cleveland Clinic Foundation, \\ Cleveland, Ohio
}

\begin{abstract}
The management of cervical spondylosis has evolved over the past several decades. Surgical decompressive and stabilization techniques have become more widely accepted for use in patients with intractable pain or neurological deficits. Advances in neuroimaging, surgical technique, and surgery-related technology including the operating microscope and anterior fixation devices have all contributed to the expanding role of surgery for the treatment of this condition. In this paper the author will focus on the role of corpectomy as a surgical option for managing cervical spondylosis.
\end{abstract}

KEY WORDS - cervical spine • spondylosis • corpectomy - anterior cervical fusion

Cervical spondylosis is the most frequent pathological condition affecting the adult spine. It is the most common cause of cervical radiculopathy and myelopathy in patients older than 50 years of age. The spondylotic process represents a spectrum of degenerative changes involving the intervertebral discs and the vertebral bodies that results in the formation of osteophytes. The development of these osteophytes can lead to the subsequent compression of adjacent nerve roots or the spinal cord, inducing a wide range of clinical signs and symptoms. When osteophytic compression of neural elements produces symptoms that are not alleviated by conservative measures, decompressive surgery may be indicated.

The surgical management of cervical spondylosis has evolved significantly over the past several decades. Initially, laminectomy was the sole option for decompressing the cervical spinal cord and nerve roots, despite the fact that cervical spondylosis-induced epidural compression most frequently occurred anterior to the neural elements. This limitation may have resulted in less than optimum clinical outcomes as a result of incomplete decompression of the neural elements.

The development of the anterior cervical approach as well as a variety of internal fixation devices gradually expanded the options available for the surgical management of cervical spondylosis. Anterior cervical surgery now represents one of the most frequently performed spinal procedures. Although a majority of these procedures

Abbreviations used in this paper: PLL = posterior longitudinal ligament; SSEP = somatosensory evoked potential. are localized to the intervertebral disc space, removal of a vertebra or several vertebrae may be indicated if the site of epidural compression extends beyond the level of a disc space. In this paper the author focuses on the indications and techniques for cervical corpectomy.

\section{SURGICAL MANAGEMENT OF CERVICAL SPONDYLOSIS}

The surgical management of cervical spondylosis remains controversial. The clinical manifestations and natural history of the disorder are variable and unpredictable. ${ }^{20,21}$ In earlier studies in which the natural history of cervical spondylosis was assessed, authors suggested this was a self-limited process..$^{7,21,26}$ Other authors have noted that surgical treatment did not alter the natural history in those patients with cervical spondylotic myelopathy. ${ }^{1,16}$

Many of these studies were limited in their assessment of clinical outcome because of the heterogeneous and often protracted course of cervical spondylosis. A comparison of the various clinical outcomes has been difficult because uniformity of several characteristics has been absent, including pathological entity, duration of follow-up period, and criteria for assessment. ${ }^{22}$ Undertaking an updated study to reassess the natural history of this disorder is difficult because of the current universal acceptance of surgical intervention for clinically significant compressive lesions of the cervical spinal cord and nerve roots.

Earlier clinical studies in which authors compared conservative management with surgical decompression were also limited by the fact that laminectomy was the primary 
surgical intervention available. Patients in whom significant anterior epidural compression was present and in whom decompressive laminectomy was performed may have undergone incomplete decompression. The residual neural compression may have compromised the clinical outcome in these patients. Furthermore, the removal of the posterior elements may have led to the development of a kyphotic cervical deformity, which compounded the anterior epidural compression.

The conclusions drawn in these studies were also shaped by the absence of several key technological and surgical advances, including improved neuroimaging modalities, the use of the operative microscope, and developments in anterior cervical decompressive and stabilization techniques. These advances have contributed to the refinement of the selection process as well as the surgical techniques for managing cervical spondylosis. They have also created additional management decisions for the surgeon, however, including selection of the appropriate surgical approach and determination of the extent of bone removal required to optimize the clinical outcome and the need for any fusion and fixation procedure. Each of these factors is a critical component in the surgical management of patients with cervical spondylosis.

Current surgical options for cervical spondylosis are relatively safe and effective when applied in the properly selected patient. General indications for surgery are: 1) progression of a myelopathy or focal motor deficit; 2) presence of intractable radicular pain unresponsive to conservative management; and 3) the presence of spinal instability or a symptomatic deformity. These indications are dependent on the presence of a radiographically demonstrated abnormality that correlates with clinical findings.

An anterior approach to the cervical spine is indicated when the major compressive disease lies ventral to the epidural space (Fig. 1 upper left). The advantage of the anterior approach is that it provides direct access to osteophytes lying ventral to the epidural space by allowing resection of one or several intervertebral discs or vertebrae. The anterior approach also allows the surgeon to fuse the affected spinal levels, thus helping to control the progression of the spondylotic process.

The disadvantage of the anterior approach is that it offers limited access to the upper cervical region, the cervicothoracic junction, or any posterior compressive disease. The anterior approach is also associated with a slightly higher surgery-related morbidity rate compared with the posterior approach. ${ }^{30}$ This higher rate of morbidity generally relates to dissection and retraction injuries involving the complex soft-tissue anatomy of the anterior cervical region. A disadvantage of anterior cervical fusion is that the disc spaces adjacent to the fused segments may over time be subjected to increased mechanical stress. Theoretically, this stress may accelerate degenerative changes in these discs and result in the development of symptoms. ${ }^{12}$

A posterior approach is indicated when there is epidural compression secondary to infolding of the ligamentum flavum, far-lateral compression of a nerve root within its foramen, or a concentrically narrowed spinal canal, provided there is neither a kyphotic deformity nor a marked

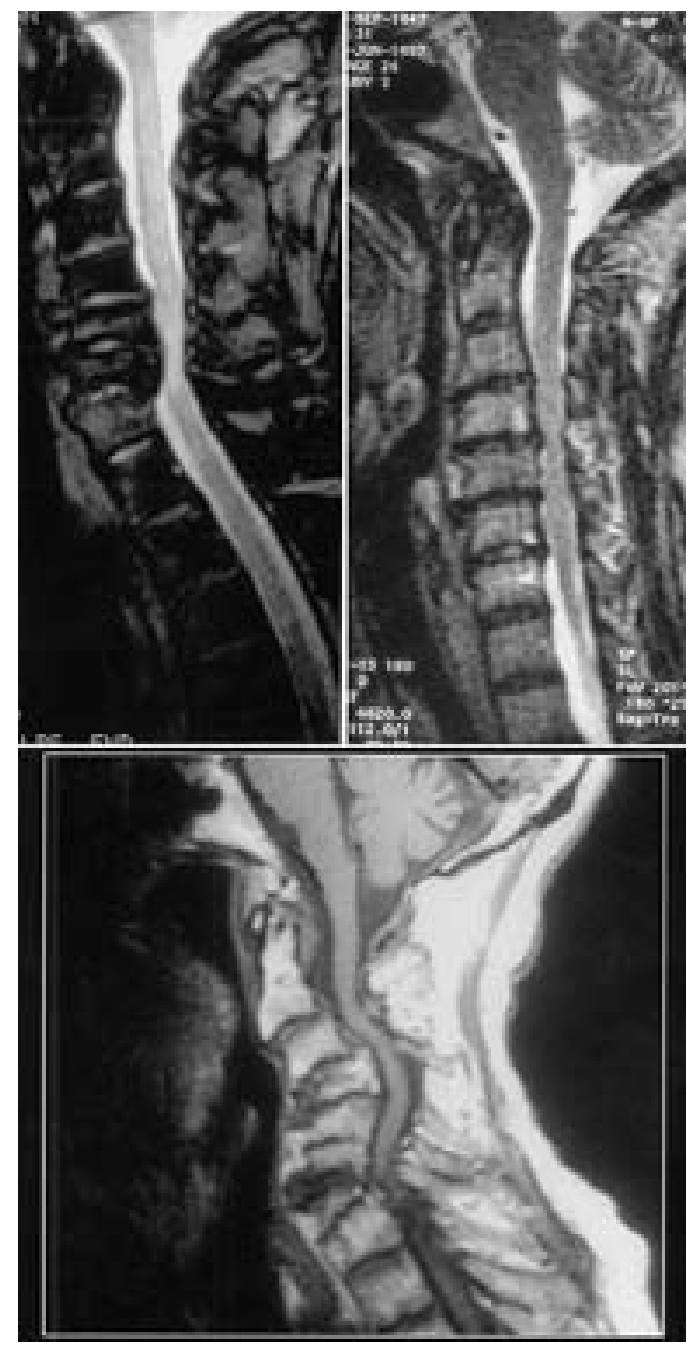

Fig. 1. Sagittal magnetic resonance images. Upper Left: Image demonstrating anterior epidural compression at C-5 and C-6. A C5-6 corpectomy and reconstruction was performed. Upper Right: Image demonstrating concentric canal stenosis. A C4-6 laminectomy was performed. Lower: Image demonstrating combined anterior and posterior epidural compression with spinal deformity. This patient underwent a combined anterior-posterior decompression and fixation.

loss of the cervical lordotic curvature (Fig. 1 upper right). Although the posterior approach is associated with a lower incidence of soft-tissue complications compared with the anterior approach, a slightly higher incidence of neurological complications and a greater potential for the development of a postdecompression deformity have been reported. ${ }^{30}$

Occasionally, both an anterior and a posterior approach may be necessary to optimize the decompression or stabilization of the spondylotic cervical spine. The combined anterior-posterior approach is usually indicated when there is a marked deformity of the cervical spine in conjunction with significant epidural compression (Fig. 1 lower). It may also be indicated after an extensive anterior decompression involving the removal of several vertebrae has been performed. 


\section{Role of corpectomy in cervical spondylosis}

The primary goals of the surgical management in patients with cervical spondylosis include the reduction of pain and the stabilization or improvement of neurological deficits, which are achieved by performing an adequate decompression of the neural elements and by preserving or restoring spinal stability. These two factors are closely linked, and the failure to address adequately either one may result in a suboptimum clinical outcome. The variety of surgical procedures now available to the spine surgeon allows one to undertake a tailored surgical approach in each patient based on the location and severity of the compressive lesion(s), the nature and degree of any spinal instability, and the radiographic appearance of the adjacent spinal elements.

\section{INDICATIONS FOR CERVICAL CORPECTOMY}

Compression of the anterior epidural space localized to the level of the individual disc spaces(s) can be sufficiently managed by performing an anterior cervical discectomy procedure. When the extent of the epidural compression extends beyond the level of the disc space(s), however, removal of the adjacent vertebrae may be necessary to achieve an adequate decompression of the neural elements.

Cervical corpectomy may be performed from C-3 to C-7. Although it usually involves the excision of only a single vertebra along with its adjacent disc spaces, the corpectomy can be extended to include four vertebrae if necessary. Typically it is combined with a reconstructive procedure that involves the insertion of a strut graft or prosthetic device into the corpectomy defect. This construct may then be further secured by placing an internal fixation device.

\section{TECHNIQUE FOR CERVICAL CORPECTOMY}

\section{Preoperative Preparation}

Several general principles apply to the treatment of all patients undergoing cervical corpectomy for spondylotic myelopathy. The positioning of the patient's head and neck during the procedure is an important preoperative consideration. Excessive extension or flexion of the cervical spine may exacerbate the epidural compression and cause a focal cord injury. A neutral or slightly extended position of the patient's cervical spine is preferable. In cases of severe epidural compression, it may be necessary to intubate the patient under fiberoptic guidance to minimize extension of the cervical spine.

The administration of corticosteroid medication is optional. In selected patients in whom severe neurological impairment has been caused by significant neural compression, preoperative administration of corticosteroids may protect the spinal cord during surgery, although limited data exist to support this strategy. Additionally, there is conflicting evidence regarding the routine use of SSEP monitoring during surgery for cervical spondylosis. Although advocates of monitoring claim a potential reduction in postoperative neurological deficits when SSEP monitoring is used, no definitive, prospective study has been performed to substantiate this claim. Because the risk of neurological deterioration related to anterior cervi- cal surgery is relatively low in the absence of monitoring, one must question whether the additional time and expense involved justifies the routine use of this intraoperative modality. Furthermore, the same degree of caution in decompressing the neural elements is used regardless of whether SSEP monitoring is included. Monitoring of SSEPs, however, may be helpful in isolated cases in which correction of a significant cervical deformity is also planned. Its use should be determined by each surgeon's personal preference and comfort level with anterior cervical surgery.

A final consideration, particularly in elderly patients with severe cord compression, is the avoidance of a sustained period of hypotension. This is particularly critical during induction of anesthesia when a brief period of transient hypotension may occur, increasing the potential for hypoperfusion of an already-compromised spinal cord. The anesthesiology team should be alerted to the potential for this problem and take the necessary precautions to avoid any extended hypotensive episode.

\section{Surgical Technique}

Although a thorough understanding of the anatomy of the anterior cervical region is beyond the scope of this review, it is critical to the success of the anterior cervical approach. The anatomical complexity of this region contributes to the technical difficulty of the approach, as well as to the variety of surgery-related complications that can occur.

The anterior approach for cervical corpectomy can be performed via a transverse incision in the anterior cervical triangle or via an oblique incision made along the medial border of the sternocleidomastoid muscle. When fully healed, the appearance of the transverse incision is cosmetically superior to that of the oblique incision. To obtain an extensive exposure of the anterior cervical spine, the transverse incision can be lengthened to provide as much exposure as that afforded by an oblique incision. Access from C-2 to T-1 can be achieved via a transverse incision.

Although a right-sided approach is technically easier for a right-handed surgeon, the left-sided approach to the cervical spine may offer some minimal reduction to the risk of injuring the recurrent laryngeal nerve. The recurrent laryngeal nerve on the right side lies between the trachea and esophagus but can take an aberrant course and lie more rostral than the nerve on the left side. ${ }^{6}$ This anatomical variation may make the nerve more prone to a retraction-related injury in cases of a right-sided approach. Alternatively, in a left-sided approach there is the potential of injuring the thoracic duct.

An avascular plane is developed between the sternocleidomastoid muscle and carotid artery laterally and the trachea and esophagus medially. The carotid artery should be palpated early in this dissection to ensure its lateral retraction. Palpation can be used to identify the cervical spine and the prevertebral fascia swept aside using a cottonoid pledget. Orientation to the vertebral midline is obtained by noting the position of the medial borders of the two longus colli muscles. A spinal needle is placed into the most rostral, accessible disc space, and a lateral radiograph is obtained to confirm the appropriate spinal level. 
After satisfactory exposure and localization, the medial borders of the longus colli muscles are elevated. This dissection should extend beyond the rostral and caudal ends of the proposed decompression site. The added length of this dissection will allow for optimum seating of a selfretaining retractor, limiting the pressure placed on the surrounding soft-tissue structures lying more anterior. The lateral dissection of the longus colli at any one level, however, should be limited to minimize the potential of inflicting an injury on the underlying vertebral artery. Excessive lateral dissection of the longus colli muscles may also result in injury to the adjacent sympathetic chain, leading to development of a unilateral Horner syndrome. ${ }^{13}$ Selfretaining retractor blades are placed beneath the medial aspects of the longus colli muscles. A second set of retractors can be positioned perpendicular to the first two blades to allow rostrocaudal retraction (Fig. 2).

The disc spaces adjacent to the vertebra to be removed are identified and partial discectomies are performed. It is critical to identify the neurocentral joints of Luschka at each disc level because they serve as important landmarks for maintaining a proper orientation to the midline as well as delineating the approximate lateral margins of the corpectomy trough. The trough should be oriented perpendicular to the vertebral endplates at each end and parallel to the adjacent longus colli muscles.

The initial bone excision (anterior half of each vertebra) can be performed using a bone rongeur. This bone can be saved and used later during the reconstruction and fusion process. The remainder of the bone resection is performed using a high-speed drill, preferably under loupe magnification or an operative microscope (Fig. 3). The drill should not be used on any bone or osteophyte that is freely

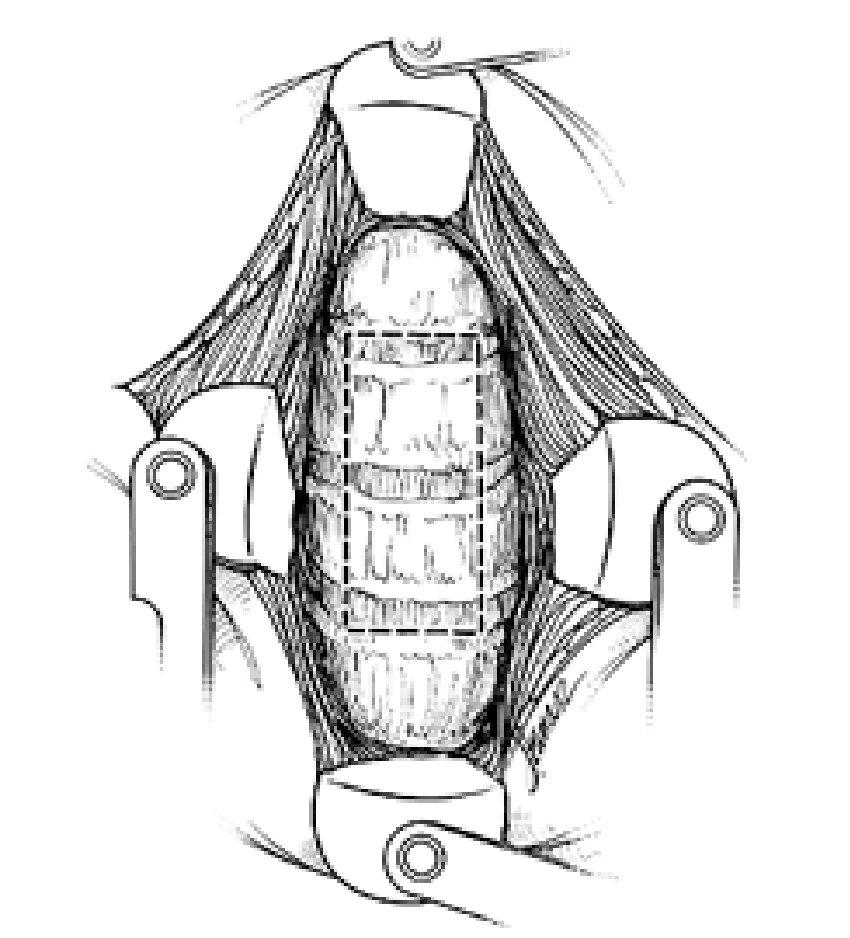

Fig. 2. Illustration demonstrating anterior exposure of the cervical spine. The extent of planned two-level corpectomy is outlined.

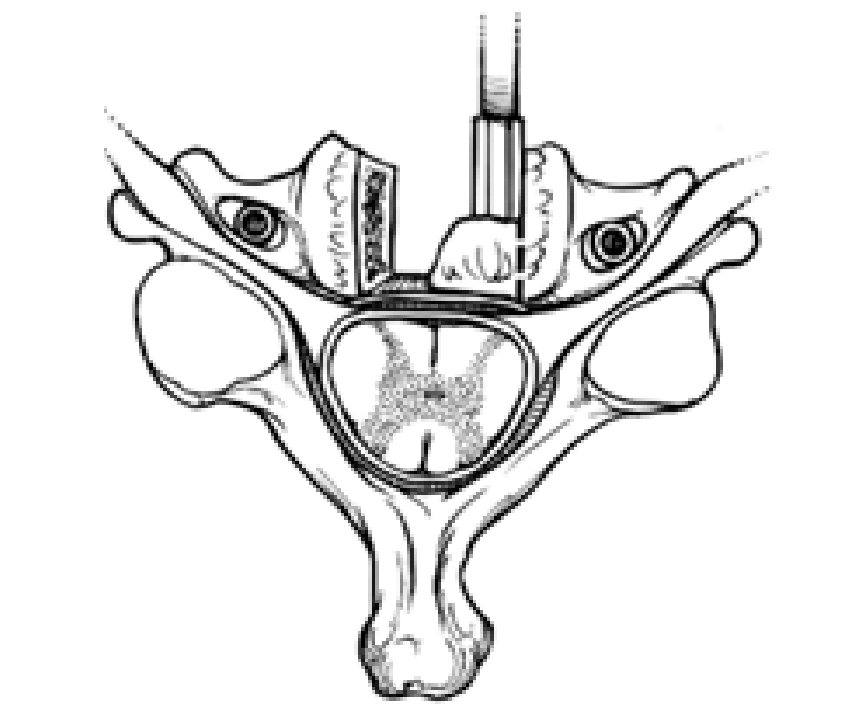

Fig. 3. Illustration depicting an axial view of the cervical spine demonstrating the width of corpectomy trough, which is typically 15 to $16 \mathrm{~mm}$.

movable because this may result in undue pressure placed on the dura mater and the underlying spinal cord.

After adequate bone removal and shaping of the corpectomy trough have been conducted, the PLL can be opened to expose the dura. Although resection of the PLL is not absolutely necessary, its removal allows for a more complete decompression of the epidural space by providing access to osteophytes attached to the two vertebrae on either end of the corpectomy trough. Additionally, lateral decompression of the exiting nerve roots within their foramen is more effectively achieved following removal of the PLL.

A sharpened nerve hook can be used to make an initial opening through the PLL. A 1-mm Kerrison punch is then used to incise the lateral margins of the PLL. The punch should not be directed toward the midline because this may further compress an already compromised spinal cord. Instead, by makiing the incision along both lateral margins of the PLL and crossing the midline with the punch at the less stenotic ends of the corpectomy trough, the PLL can frequently be excised as a single section, further limiting the potential for iatrogenic injury to the spinal cord.

When completed, the corpectomy trough should measure approximately 15 to $16 \mathrm{~mm}$ in width. This ensures a thorough decompression across the entire epidural space. The apex of the dural curvature as it extends from one side of the decompression to the other should be centered within the trough. A dural guide should be easily passed beneath both ends vertebrae if the PLL has been removed.

After the corpectomy is completed, a strut bone graft is prepared and positioned. The two most common options for a strut graft in the cervical spine are a fibular or iliac crest graft. Although an iliac crest graft has more cancellous bone to help facilitate early graft incorporation, a fibular graft has more cortical bone to provide early structural support. Despite these differences, both grafts are highly effective for postcorpectomy reconstruction. Al- 
ternatively, a titanium mesh or carbon fiber cage material can be used for vertebral reconstruction.

The issue of whether to use autograft or allograft is not as significant in cases of anterior cervical surgery as it is in those involving posterior fusion procedures. Bone graft incorporation occurs more readily with the graft under compression (anterior fusion) than it does with the graft under tension (posterior onlay fusion). ${ }^{34}$ For this reason, either an autograft or an allograft may be used as an anterior strut graft. Although an autograft is associated with an earlier remodeling and incorporation rate, use of an allograft avoids the complications associated with harvesting an iliac crest or fibular autograft. ${ }^{18,29,36}$ These complications include persistent pain at the harvest site, paresthesias in the distribution of the related peripheral nerves, vascular injury, adjacent bone fracture (pelvic crest), and wound infection. ${ }^{19}$ Although the use of an allograft is associated with the theoretical risk for disease transmission, the current procurement and sterilization techniques for acquiring bone allograft virtually eliminate this risk. ${ }^{5,29}$

A third option for postcorpectomy reconstruction is a strut graft in which both autograft and allograft are used. This procedure involves filling the hollow center of a fibular allograft with local autograft harvested from the corpectomy site. The strut graft has the advantage of providing autograft bone for the reconstruction without the disadvantage of potential harvesting-related complications.

After selection and adequate sizing and shaping, the graft may be positioned and secured using two different methods. One technique involves creating notches in the vertebral endplates at one or both ends of the corpectomy trough. The end(s) of the graft are then cut so that they seat deeply with the notched endplates (Fig. 4 left). This may require additional bone removal from the vertebrae at each end of the trough to create the notches. Furthermore

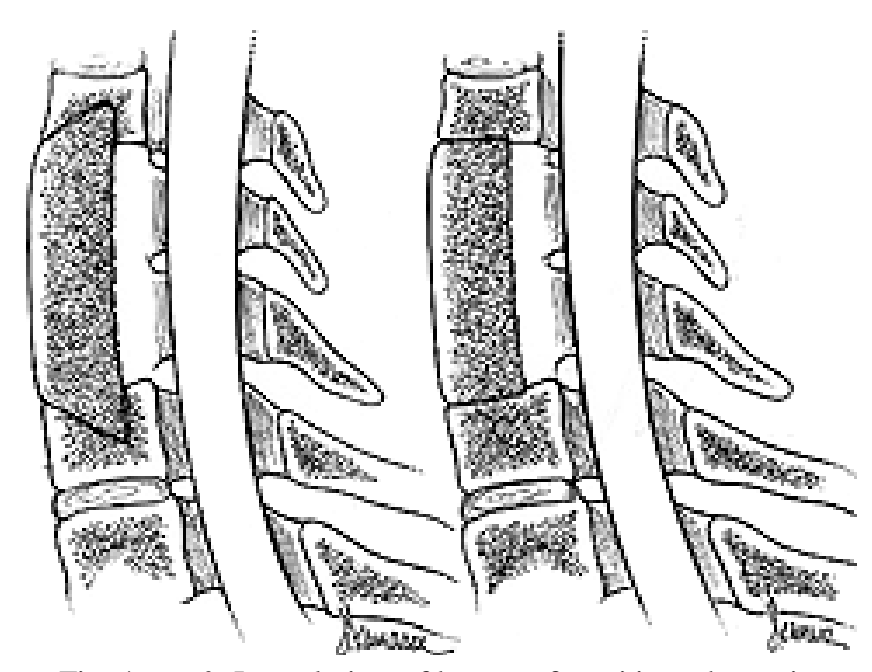

Fig. 4. Left: Lateral view of bone graft position when using a mortising technique. Note the notches cut into the endplates at the two vertebral ends. This allows for a settling of the graft that may minimize the risk of graft dislodgment. Right: Lateral view of bone graft positioning when not using mortising technique. The graft is cut 3 to $4 \mathrm{~mm}$ longer than the length of the corpectomy trough and inserted during cervical traction. The friction fit obtained minimizes the risk of graft dislodgment. this may result in a delayed subsidence of the graft into the adjacent vertebrae because of the apposition of the harder cortical bone of the graft with the softer cancellous vertebral bone. It may also require a longer strut graft, which may limit the use of an autograft or anterior plate fixation.

Alternatively, the strut graft can be positioned without the use of notches. The two vertebral endplates forming the superior and inferior margins of the corpectomy trough are prepared so that they are perpendicular to the dura and parallel to each other. The graft is cut so that its two ends are also parallel to each other and its length slightly longer $(3-4 \mathrm{~mm})$ than the length of the trough. The graft is positioned under cervical traction or distraction if distraction posts have been placed into the two end vertebrae. When properly fashioned, a friction fit is achieved that is secure enough to keep the graft in place, particularly when combined with anterior plate fixation (Fig. 4 right). This allows for the use of a shorter-length graft and minimizes the potential for graft subsidence because the cortical endplates of the adjacent vertebrae remain intact. Provided that the cartilaginous surface of the vertebral endplates are removed and good bone "carpentry" techniques are used, there is a high rate of graft incorporation associated with this technique.

\section{Anterior Cervical Fixation}

The development of standardized anterior cervical fixation systems has popularized the use of internal fixation to facilitate the bone fusion process following cervical corpectomy. By providing immediate internal stability, anterior fixation offers the potential advantages of increasing bone fusion rates, decreasing the rate of graft collapse, reducing the potential for the development of a kyphotic deformity, and minimizing the need for postoperative external immobilization. . $^{4,17,28,33}$

A number of anterior cervical fixation systems have been developed over the past decade. Each requires the selection and contouring of an appropriately sized titanium plate or dual rod component, which can then be secured, using cancellous bone screws, to the vertebrae immediately above and below the graft. The plates may also be used to secure a bone graft in position. This can be done by placing a screw(s) through the plate into the graft or by using a heavy suture to tie the graft to the plate.

Earlier plate systems required that the screws engage both the anterior and the posterior cortices of the vertebral body, which necessitated the use of intraoperative fluoroscopy to assess accurately the positioning of the near the epidural space. With the development of locking mechanisms that secure the screws to the plate and minimize screw backout, current fixation systems require only unicortical screw engagement in the vertebra (Fig. 5). This makes the use of intraoperative fluoroscopy for placing anterior cervical fixation optional.

Current anterior fixation systems also have varying degrees of implant-related rigidity. The rigidity of these systems can be classified as either constrained or nonconstrained. In a constrained system the implant is a relatively rigid screws that are inserted at a fixed angle into the vertebrae without any movement at the screw-plate interface. A nonconstrained system is biomechanically less rigid than a constrained system. In general, the screws can 


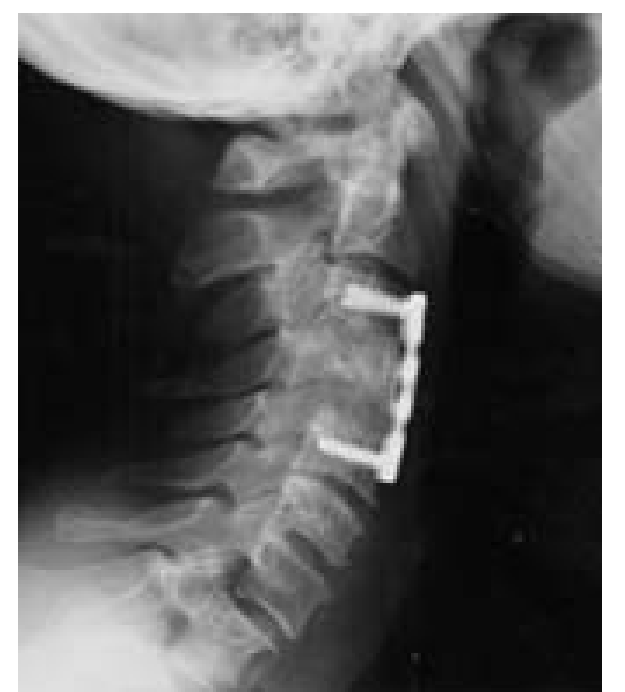

Fig. 5. Lateral radiograph revealing satisfactory graft placement and anterior plate fixation following a C-4 corpectomy.

be inserted at varied angles and the screw-implant interface is not rigidly fixed. The screws in a nonconstrained system may toggle within the vertebrae as an axial load is applied. Alternatively, the screws may be permitted to glide or translate within the implant as a load is applied. The theoretical advantage of a nonconstrained system is that it allows for a gradual transfer of forces from the implant to the bone graft. This gradual load transfer onto the graft theoretically facilitates the fusion process by minimizing bone resorption associated with the stressshielding property of a rigid system. ${ }^{18,25}$

Although anterior cervical fixation offers several theoretical advantages, the placement of a rigid device following multilevel corpectomy has been questioned. Foley, et al., ${ }^{11}$ investigated the in vitro effects of rigid anterior cervical fixation on multilevel strut graft constructs. They performed biomechanical testing in cadaver cervical spine specimens following a three-level corpectomy and strut graft reconstruction. In the group of scecimens not fitted with instrumentation, the load placed on the anterior strut graft was increased in cervical flexion and decreased in extension. When a rigid plate was applied to the specimens, the loading pattern of the graft was reversed, and an excessive graft load was observed in cervical extension. Although all plate-fitted specimens significantly decreased the local motion at C3-7 compared with the harvested spine, the excessive loads placed on the graft with even small degrees of extension approached the failure strength of the adjacent endplates. The authors concluded that applying such a construct after multilevel corpectomy could increase the potential for graft subsidence or "pistoning" into the adjacent vertebrae with subsequent failure of the construct.

In addition to anterior cervical fixation, posterior instrumentation alone or in combination with an anterior device can be used following multilevel corpectomy. ${ }^{24}$ When a combined approach is used, the addition of posterior instrumentation counteracts the individual tension band effect (describing the effect that the anterior plate has on the cervical spine) of the anterior plate, reducing the motion- related loads placed on the graft. This subsequently reduces the potential for construct failure in the three- and four-level corpectomy model.

After satisfactory positioning of the graft or prosthetic device, an implant of appropriate length is selected. The holes on each end of the implant should extend just beyond each end of the graft. This allows for a greater degree of bone access for the screws in the two terminal vertebrae and also minimizes the risk that a screw will be placed into an adjacent disc space. The implant should not span an intact disc space, because this may lead to excessive motion in the construct and subsequent loosening of the screws and plate.

After wound closure, the patient wears a hard cervical collar for 8 to 12 weeks. Halo brace immobilization is rarely necessary. In the setting of a three- or four-level corpectomy, supplemental posterior fixation is usually performed to reduce the higher morbidity rate frequently demonstrated in cases in which the longer anterior constructs are used. Radiographs are obtained immediately postoperatively and at regular intervals (3-6 months) for a period of 2 years to assess graft incorporation and fixation competence.

Although internal fixation devices have provided a significant advance in the field of anterior cervical spine surgery, they should not be considered a substitute for sound bone grafting techniques. The role of these devices is to provide immediate, rigid immobilization to facilitate early and solid bone graft incorporation. Without a solid fusion, repetitive mechanical forces may ultimately lead to device-related failure in the form of screw backout or fracturing of the implant or screws. Device-related failure, in turn, will increase the potential for pseudarthrosis and clinically significant segmental instability.

\section{COMPLICATIONS}

A wide variety of complications can occur as a result of cervical corpectomy procedures. Saunders, et al. ${ }^{31}$ have reported a series of 40 patients undergoing corpectomy in which the perioperative complication rate was $47.5 \%$. Most of these complications were secondary to the softtissue exposure required for the corpectomy. Inadequate release of fascial tissue planes can result in damage to the esophagus, trachea, carotid or vertebral arteries, or the recurrent laryngeal nerve. Injury to the spinal cord or nerve roots is less frequent, but an incomplete decompression of the neural elements is more likely than with anterior cervical discectomy is performed. When a neural injury does occur during decompression, it most commonly involves the C-5 nerve root and is usually transient. ${ }^{31}$

More common in cervical corpectomy are those complications related to bone grafting. ${ }^{2,3,10,15}$ Although the rate of pseudarthrosis is higher with allograft than autograft, this difference can be minimized by performing careful and meticulous bone grafting techniques and by the use of internal immobilization. Additional bone graft complications are related to the length of the grafts used and the forces acting upon them. The longer the strut graft, the greater the degree of leverage placed on the graft, particularly at the caudal end during cervical extension. For this reason, longer-length grafts have a greater potential for dislodgment. Although anterior fixation devices may min- 


\section{Role of corpectomy in cervical spondylosis}

imize this risk, they too are subjected to the same forces of leverage placed on the longer strut grafts. If these devices are not adequately secured, they may loosen and pull the graft out of position.

Bone graft-related complications are limited by meticulous preparation of the graft site and selection of an appropriate bone graft. Preparation of the graft site involves removal of the cartilaginous surfaces of the adjoining endplates or precise mortising of the endplates and the graft. An adequate period of postoperative cervical immobilization is also necessary to ensure a successful fusion.

Soft-tissue complications related to cervical corpectomy can be minimized by careful anterior exposure and adequate release of all tissue planes over the cervical spine. Secure positioning of self-retaining retractors will further minimize the risk of soft-tissue injury. When in place, the retractors should be intermittently released to limit the effect of long-term pressure on the adjacent anatomical structures.

\section{CLINICAL OUTCOME OF CERVICAL CORPECTOMY}

The authors of several clinical studies have evaluated the outcome of patients who have undergone cervical corpectomy for spondylotic myelopathy. Ebersold, et al., ${ }^{9}$ noted immediate clinical improvement in $23(70 \%)$ of 33 patients; the mean improvement in Nurick grade was 1.5 after cervical corpectomy. Clinical status in nine patients was unchanged and deterioration occurred in one case at early follow up. At long-term follow up (mean 6.96 years) neurological status in 18 patients $(54.5 \%)$ remained improved, was unchanged in nine $(27.3 \%)$, and had deteriorated in six $(18.2 \%)$. They found that age, severity of disease, number of levels decompressed, and preoperative Nurick grade were not predictive of outcome. The only factor related to potential deterioration was the duration of symptoms present preoperatively.

Macdonald, et al., ${ }^{23}$ analyzed their results in 36 patients who underwent cervical corpectomy followed by fibular allograft reconstruction. Myelopathy improved in 17 patients, whereas in 15 patients if remained unchanged and in four patients it deteriorated, based on the Nurick classification. They reported a surgery-related morbidity or permanent major morbidity rate of $22 \%$ (eight patients).

In a review of several other studies in which authors evaluated the results of cervical corpectomy, we found similar results. Clinical improvement in myelopathy was noted in 73 to $100 \%$ of patients..$^{2-4,14,27,32,33,35}$ Although each of these studies was designed as a retrospective review, they nonetheless indicate an improvement in the natural history of cervical spondylosis in patients who undergo anterior decompressive surgery. The mean morbidity rate of $31 \%$ emphasizes the fact that cervical corpectomy can be a technically challenging procedure with a potentially high morbidity rate.

\section{CONCLUSIONS}

Advances in surgical techniques and spinal stabilization methods have expanded the role of corpectomy for the management of cervical spondylotic myelopathy. In the properly selected individual, it allows for a direct decompression of the anterior epidural space and stabilization of the appropriate spinal motion segments. When extensive multilevel decompression is required, however, cervical corpectomy can be a technically demanding procedure that can result in a wide variety of perioperative complications. The procedure requires a precise evaluation of the individual decompression-related requirements as well as a thorough knowledge of the related cervical anatomy, the pertinent spinal biomechanics, and general techniques of anterior cervical spine surgery.

\section{References}

1. Arnasson O, Carlsson CA, Pellettieri L: Surgical and conservative treatment of cervical spondylotic radiculopathy and myelopathy. Acta Neurochir 84:48-53, 1987

2. Bernard TN Jr, Whitecloud TS III: Cervical spondylotic myelopathy and myeloradiculopathy. Anterior decompression and stabilization with autogenous fibula strut graft. Clin Orthop 221:149-160, 1987

3. Boni M, Cherubino P, Denaro V, et al: Multiple subtotal somatectomy. Technique and evaluation of a series of 39 cases. Spine 9:358-362, 1984

4. Brown JA, Havel P, Ebraheim N, et al: Cervical stabilization by plate and bone fusion. Spine 13:236-240, 1988

5. Buck BE, Malinin TI, Brown MD: Bone transplantation and human immunodeficiency virus. An estimate of risk of acquired immunodeficiency syndrome (AIDS). Clin Orthop 240: 129-136, 1989

6. Bulger RF, Rejowski JE, Beatty RA: Vocal cord paralysis associated with anterior cervical fusion: considerations for prevention and treatment. J Neurosurg 62:657-661, 1985

7. Campbell AMG, Phillips DG: Cervical disc lesions with neurological disorder, Differential diagnosis, treatment, and prognosis. Br Med J 2:481-485, 1960 (Reference unverified)

8. Caspar W: Anterior stabilization with trapezoid osteosynthetic technique in cervical spine injuries, in Kehr P, Weidnner A (eds): Cervical Spine. New York: Springer-Verlag, 1987, pp 198-204 (Reference unverified)

9. Ebersold MJ, Pare MC, Quast LM: Surgical treatment for cervical spondylitic myelopathy. J Neurosurg 82:745-751, 1995

10. Fernyhough JC, White JI, LaRocca H: Fusion rates in multilevel cervical spondylosis comparing allograft fibula with autograft fibula in 126 patients. Spine 16 (Suppl 10):S561-S564, 1991

11. Foley KT, DiAngelo DJ, Rampersaud YR, et al: The in vitro effects of instrumentation on multilevel cervical strut-graft mechanics. Spine 24:2366-2376, 1999

12. Goto S, Mochizuki M, Watanabe T, et al: Long-term follow-up study of anterior surgery for cervical spondylotic myelopathy with special reference to the magnetic resonance imaging findings in 52 cases. Clin Orthop 291:142-153, 1993

13. Graham JJ: Complications of cervical spine surgery, in Cervical Spine Research Society Editorial Committee (ed): The Cervical Spine, ed 2. Philadelphia: Lippincott, 1989, pp 831-837 (Reference unverified)

14. Hanai K, Fujiyoshi F, Kamei K: Subtotal vertebrectomy and spinal fusion for cervical spondylotic myelopathy. Spine 11: 310-315, 1986

15. Herkowitz HN: The surgical management of cervical spondylotic radiculopathy and myelopathy. Clin Orthop 239:94-108, 1989

16. Hunt WE: Cervical spondylosis; natural history and rare indications for surgical decompression. Clin Neurosurg 27:466-480, 1980

17. Kalfas IH: The anterior cervical spine locking plate: a technique for surgical decompression and stabilization, in Fessler RG, 
Haid RW (eds): Techniques in Spinal Stabilization. New York: McGraw-Hill, 1996, pp 25-33 (Reference unverified)

18. Kaufman HH, Jones E: The principles of bony spinal fusion. Neurosurgery 24:264-270, 1989

19. Kurz LT, Garfin SR, Booth RE Jr: Harvesting autogenous iliac bone grafts. A review of complications and techniques. Spine 14:1324-1331, 1989

20. LaRocca H: Cervical spondylotic myelopathy: natural history. Spine 13:854-855, 1988

21. Lees F, Turner JWA: Natural history and prognosis of cervical spondylosis. Br Med J 2:1607-1610, 1963 (Reference unverified)

22. Lunsford LD, Bissonette DJ, Zorub DS: Anterior surgery for cervical disc disease. Part 2: Treatment of cervical spondylotic myelopathy in 32 cases. J Neurosurg 53:12-19, 1980

23. Macdonald RL, Fehlings MG, Tator CH, et al: Multilevel anterior cervical corpectomy and fibular allograft fusion for cervical myelopathy. J Neurosurg 86:990-997, 1997

24. McAfee PC, Bohlman HH, Ducker TB, et al: One-stage anterior cervical decompression and posterior stabilization. A study of one hundred patients with a minimum of two years of followup. J Bone Joint Surg Am 77:1791-1800, 1995

25. McAfee PC, Farey ID, Sutterlin CE, et al: 1989 Volvo Award in basic science. Device-related osteoporosis with spinal instrumentation. Spine 14:919-926, 1989

26. Nurick $S$ : The natural history and the results of surgical treatment of the spinal cord disorder associated with cervical spondylosis. Brain 95:101-108, 1972

27. Okada K, Shirasaki N, Hayashi H, et al: Treatment of cervical spondylotic myelopathy by enlargement of the spinal canal anteriorly, followed by arthrodesis. J Bone Joint Surg Am 73: 352-364, 1991

28. Papadopoulos SM, Kalfas IH, Sonntag VKH: Anterior Instru- mentation of the Cervical Spine, in Whitecloud TS III, Dunsker SB (eds): Anterior Cervical Spine Surgery. New York: Raven Press, 1993, pp 89-103 (Reference unverified)

29. Prolo DJ: Biology of bone fusion. Clin Neurosurg 36: 135-146, 1988

30. Raynor RB: Anterior and posterior approaches to the cervical spinal cord, discs, and roots: a comparison of exposures and decompression, in Cervical Spine Research Society Editorial Committee (ed): The Cervical Spine, ed 2. Philadelphia: Lippincott, 1989, pp 659-669 (Reference unverified)

31. Saunders RL, Bernini PM, Shirreffs TG Jr, et al: Central corepectomy for cervical spondylotic myelopathy: a consecutive series with long-term follow-up evaluation. J Neurosurg 74: 163-170, 1991

32. Seifert V, Stolke D: Multisegmental cervical spondylosis: treatment by spondylectomy, microsurgical decompression, and osteosynthesis. Neurosurgery 29:498-503, 1991

33. Tippets RH, Apfelbaum RI: anterior cervical fusion with the Caspar instrumentation system. Neurosurgery 22:1008-1013, 1988

34. White AA III, Panjabi MM: Clinical Biomechanics of the Spine, ed 2. Philadelphia: Lippincott, 1990

35. Zdeblick TA, Bohlman HH: Cervical kyphosis and myelopathy. Treatment by anterior corpectomy and strut-grafting. J Bone Joint Surg Am 71:170-182, 1989

36. Zdeblick TA, Ducker TB: The use of freeze-dried allograft bone for anterior cervical fusions. Spine 16:726-729, 1991

Manuscript received November 15, 2001.

Accepted in final form December 11, 2001.

Address reprint requests to: Iain H. Kalfas, M.D., Department of Neurosurgery (S80), The Cleveland Clinic Foundation, 9500 Euclid Avenue, Cleveland, Ohio 44195. 